\title{
The impact of exercise on growth factors (VEGF and FGF2): results from a 12-month randomized intervention trial
}

\author{
Darren R. Brenner ${ }^{1,2,3^{*}}$ D, Yibing Ruan ${ }^{1}$, Scott C. Adams ${ }^{4}$, Kerry S. Courneya ${ }^{4}$ and Christine M. Friedenreich ${ }^{1,2,3}$
}

\begin{abstract}
Background: Vascular endothelial growth factor (VEGF) and Fibroblast growth factor-2 (FGF2) are angiogenic cytokines in normal tissues and tumors. Evidence suggests that increased growth factor expression in adipose tissue leads to improved vascularity and decreased hypoxia, fibrosis, and inflammation, which may, in turn, reduce postmenopausal breast cancer risk.

Objective: We investigated whether or not exercise had dose-response effects on levels of plasma VEGF and FGF2 in postmenopausal women.

Methods: Four hundred previously inactive but healthy postmenopausal women aged 50-74 years of age were randomized to 150 or 300 min per week of aerobic exercise in a year-long exercise intervention. VEGF and FGF2 were measured from fasting serum samples with a custom-plex multiplex assay.

Results: A high compared to moderate volume of aerobic exercise did not cause chronic changes in plasma VEGF or FGF2 levels in intention-to-treat or per-protocol analyses.

Conclusions: We did not detect differences in growth factor levels related to increasing doses of exercise. It is unlikely that changes in VEGF and FGF2 levels mediate the reduction in risk of post-menopausal breast cancer development in associated with increased levels of exercise.
\end{abstract}

Trial registration: Clinicaltrials.gov identifier: NCT01435005.

Keywords: Aerobic exercise, Growth factors, Breast Cancer, Intervention trial, Randomized controlled trial

\section{Introduction}

Regular lifetime exercise has been consistently associated with a reduced risk of developing postmenopausal breast cancer [1]. Several mechanisms underlying the impact of exercise have been identified, including reductions in adiposity, sex hormone levels, insulin resistance and chronic inflammation [1]. These pathways explain some portion of the association; however, additional mechanisms require identification and further investigation.

One additional mechanism of interest is the impact of exercise on growth factors known to drive cell proliferation

\footnotetext{
* Correspondence: Darren.Brenner@ucalgary.ca

${ }^{1}$ Department of Oncology, Cumming School of Medicine, University of Calgary, Calgary, Alberta, Canada

${ }^{2}$ Department of Community Health Sciences, Cumming School of Medicine,

University of Calgary, Calgary, Alberta, Canada

Full list of author information is available at the end of the article
}

and tissue growth [2]. Vascular endothelial growth factor (VEGF) is a potent angiogenic cytokine in normal tissues and tumors. It has been shown to stimulate endothelial cell proliferation in vitro and induce angiogenesis in vivo [3]. Fibroblast growth factors are a family of growth factors related to a broad range of physiologic functions including angiogenesis and epithelial proliferation [4]. Fibroblast growth factor-2 (FGF2) is an interesting candidate in breast cancer pathology as a common single nucleotide polymorphism in the gene has been associated with breast cancer risk in large studies among Western populations [5].

In a previous investigation from the Breast cancer and Exercise Trial in Alberta (BETA), we observed that higher levels of activity were associated with reduced body fat [6]. Unhealthy fat pad expansion is characterized by inadequate vascularization and hypoxia - leading to increased fibrosis, inflammation and insulin resistance 
Table 1 Intention-to-treat and per-protocol analysis of FGF2 and VEGF concentrations between high and moderate volume exercise groups in BETA $(n=386)$

\begin{tabular}{|c|c|c|c|c|c|c|c|c|c|c|}
\hline \multirow[t]{2}{*}{ Group } & \multirow[t]{2}{*}{$n$} & \multicolumn{2}{|l|}{ Baseline } & \multicolumn{2}{|l|}{6 Months } & \multicolumn{2}{|l|}{12 Months } & \multicolumn{2}{|c|}{ Treatment Effect } & \multirow{2}{*}{$\begin{array}{l}\text { Between- } \\
\text { Group P }\end{array}$} \\
\hline & & Geo-metric Mean $^{a}$ & $95 \% \mathrm{Cl}$ & Geo-metric Mean & $95 \% \mathrm{Cl}$ & Geo-metric Mean & $95 \% \mathrm{Cl}$ & $\begin{array}{l}\text { Ratio of High/ } \\
\text { Moderate }\end{array}$ & $95 \% \mathrm{Cl}$ & \\
\hline \multicolumn{11}{|c|}{ Intention-to-treat } \\
\hline \multicolumn{11}{|l|}{ FGF2 } \\
\hline High & 195 & 121.3 & $114.4-128.5$ & 116.1 & $110.1-122.4$ & 123.3 & $117.7-129.2$ & 0.98 & $0.94-1.02$ & 0.28 \\
\hline Moderate & 191 & 118.8 & $111.9-126.2$ & 122.9 & $116.2-130.0$ & 120.1 & $113.1-127.6$ & & & \\
\hline \multicolumn{11}{|l|}{ VEGF } \\
\hline High & 195 & 62.3 & $55.1-70.4$ & 58.5 & $51.7-66.3$ & 58.6 & $51.3-66.9$ & 0.94 & $0.86-1.02$ & 0.15 \\
\hline Moderate & 191 & 64.9 & $57.2-73.6$ & 63.6 & $55.7-72.6$ & 67.3 & $58.9-76.9$ & & & \\
\hline \multicolumn{11}{|c|}{ Per-protocol ${ }^{d}$} \\
\hline FGF2 & & & & & & & & & & 0.64 \\
\hline High & 80 & 119.8 & $110.8-129.4$ & 116.0 & $107.3-125.3$ & 121.2 & $113.5-129.4$ & 1.02 & $0.95-1.10$ & \\
\hline Moderate & 58 & 112.6 & $100.0-126.7$ & 113.9 & $101.9-127.3$ & 109.6 & $98.3-122.2$ & & & \\
\hline VEGF & & & & & & & & & & 0.74 \\
\hline High & 80 & 60.6 & $50.8-72.3$ & 60.6 & $50.0-73.4$ & 58.0 & $47.6-70.7$ & 0.98 & $0.84-1.13$ & \\
\hline Moderate & 58 & 70.3 & $54.2-91.0$ & 64.9 & $49.9-84.6$ & 74.0 & $56.9-96.3$ & & & \\
\hline
\end{tabular}

a. Geometric mean of FGF2 and VEGF was in the unit of $\mathrm{pg} / \mathrm{mL}$

b. The geometric mean ratios were estimated from least square means for the difference in treatment effect between high and moderate volume exercisers averaged across the entire study period adjusted for the baseline values and then back log-transformed

c. $P$ value corresponds to the null hypothesis that the ratio of treatment effect between high- and moderate-volume groups equals 1 against the 2 sided alternative hypothesis

d. Women assigned to the moderate-volume group were adherent if they completed 90 to $100 \%$ of the exercise prescription (mean, 135-150 min/wk), weeks 13 to 52 at full prescription; women assigned to the high-volume group were adherent if they completed at least $90 \%$ of the exercise prescription (mean, $\geq 270 \mathrm{~min} / \mathrm{wk}$ ), weeks 13 to 52 at full prescription

[7]. We hypothesized that exercise-induced change in adipose tissue vascular remodeling may account for some of the observed exercise-related reduction in postmenopausal breast cancer risk. As part of ancillary investigations from BETA to evaluate novel mechanisms relating exercise to breast cancer risk, we investigated whether a higher versus a more moderate volume of exercise would lead to differential changes in VEGF and FGF2 levels.

\section{Materials and methods}

BETA study design and eligibility criteria have been described elsewhere [8]. Briefly, we randomized 400 postmenopausal women, aged 50-74 years old, who were previously inactive but otherwise healthy to 150 (MODERATE) or 300 (HIGH) minutes/week of aerobic exercise at $65-75 \%$ of maximal heart rate reserve for 12 months. Participants completed three supervised sessions and two unsupervised sessions per week, with the HIGH volume exercise group completely exercise sessions that were twice as long as the MODERATE volume group. During exercise sessions, any aerobic activity that raised that participants' heart rates to their target heart rate zones was permitted.

Fasting blood samples were drawn from study participants at baseline, 6 and 12 months, after a 24-h complete abstinence from exercise and alcohol intake and at least $10 \mathrm{~h}$ since the last food intake. FGF2 and VEGF levels in plasma were quantified using a customplex Human Circulating Cancer Biomarker Multiplex Assay. The multiplex assays were performed at Eve Technologies (Calgary, AB, Canada) using the Bio-Plex ${ }^{\text {Tx }}$ 200 system (Bio-Rad Laboratories, Inc., Hercules, CA, USA). The detection limits for FGF2 and VEGF in this assay were 3.6 and $6.4 \mathrm{pg} / \mathrm{mL}$, respectively. The interand intra-assay coefficients of variation (CV) were 6.3 and $7.9 \%$ for FGF2, and 10.2 and $12 \%$ for VEGF, which are below or comparable to other CVs using the BioPlex platform [9]. All lab personnel were blinded to the intervention assignment. Each participant's samples at different time points were analyzed in the same batch and each batch included an equal number of MODERATE and HIGH blood samples.

Changes in concentrations of growth factors in the two intervention groups were compared in an intentionto-treat (ITT) analysis using linear mixed models that accounted for repeated measures of FGF2 and VEGF at 6 and 12 months, adjusting for baseline levels of the biomarker as a covariate. Treatment effect ratios (TERs) were calculated from these models and denoted the HIGH: MODERATE ratio of geometric mean biomarker levels over 12 months (reflecting changes from baseline 
Brenner et al. European Review of Aging and Physical Activity

(2019) $16: 8$

Page 3 of 6

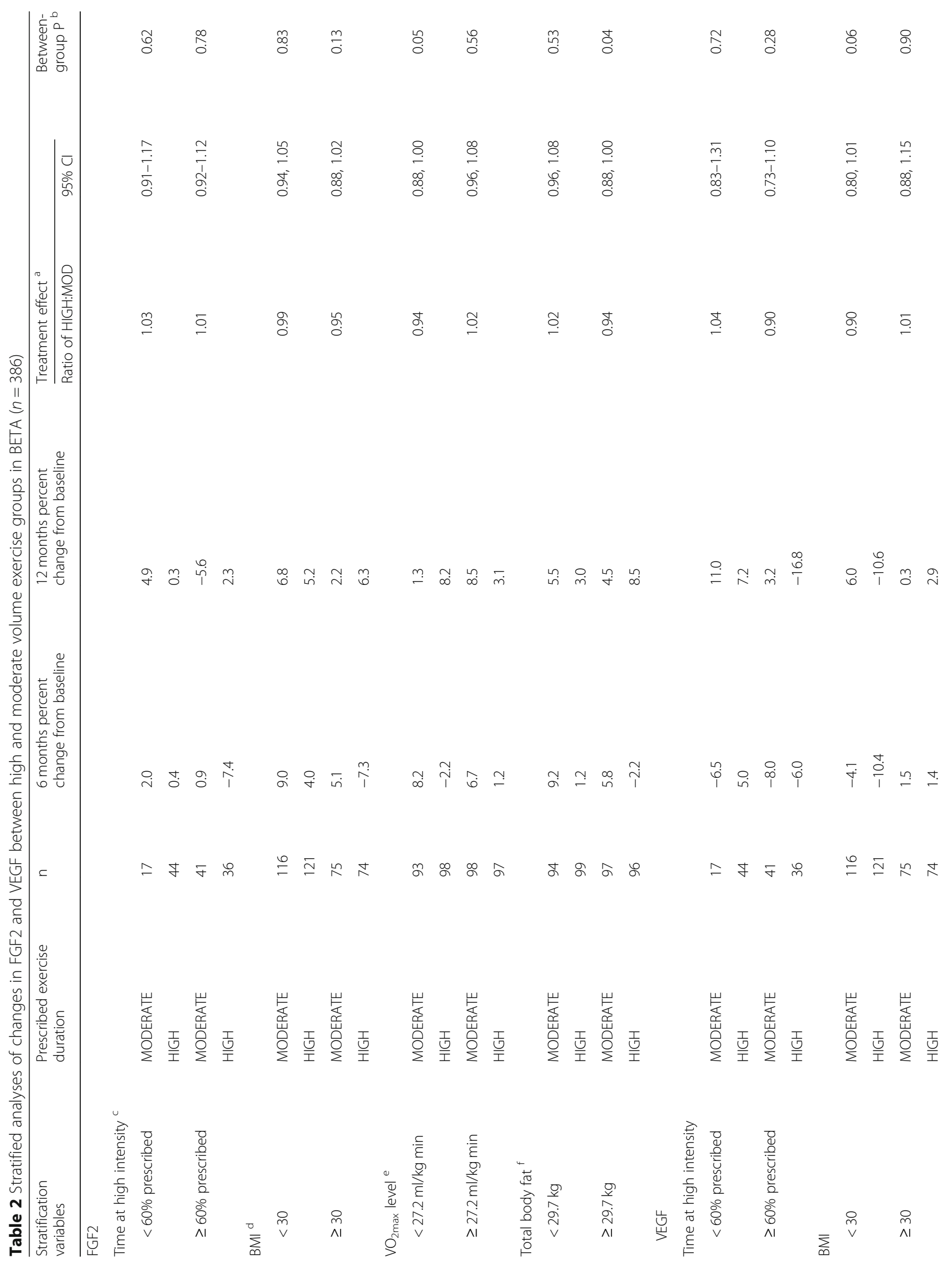




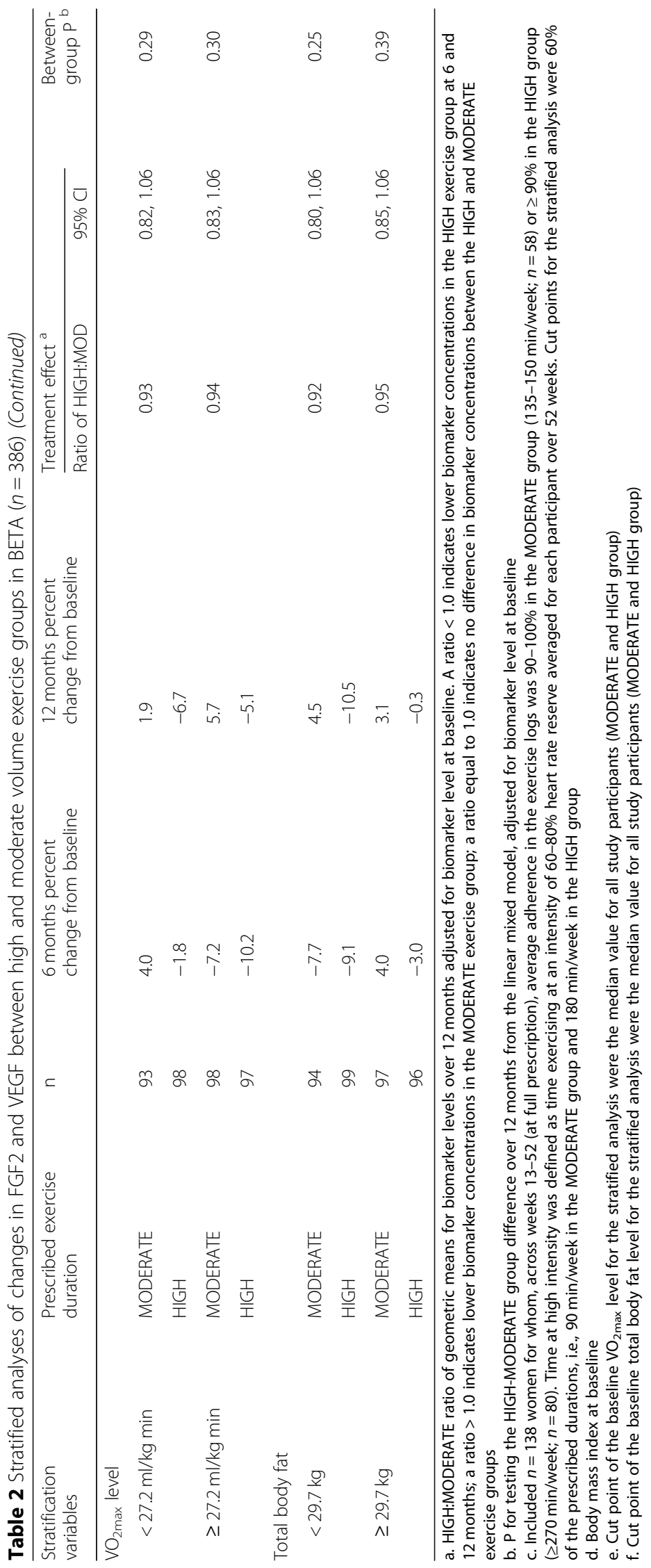


to 6 and 12 months). The ITT analysis was repeated by stratifying on a priori potential effect modifiers: baseline body mass index (BMI), baseline fitness (estimated $\mathrm{VO}_{2 \max }$ ) and total percent body fat (full body dual X-ray absorptiometry scan).

A per-protocol analysis was undertaken for women who completed, on average, 90 to $100 \%$ of the exercise prescription for the MODERATE group or $\geq 90 \%$ of prescription for the HIGH group from weeks 13-52.

\section{Results}

A total of 386 women completed the trial with no statistically significant difference in dropout between arms. Baseline characteristics and exercise adherence of the BETA study participants have been previously described [6]. We observed no statistically significant effects for increasing quartiles of exercise and change in circulating levels of VEGF or FGF2 with both groups combined (non-randomized, results not shown).

Results from linear mixed models suggested overall lower, but non-statistically significant group differences between baseline and 12 months for both FGF2 and VEGF for both arms of the trial. The HIGH group experienced slightly larger changes than the MODERATE group, but these changes were not clinically relevant (FGF2: TER 0.98, 95\% CI 0.94-1.02; VEGF: TER 0.94, 95\% CI 0.86-1.02; Table 1). Furthermore, the per-protocol analysis found essentially no differences between baseline and end of intervention effects with the TERs approximately equal to 1.0 for both biomarkers. Similarly, a per-protocol analysis stratified by exercise intensity, i.e., whether participants achieved $60 \%$ of the prescribed time in the target heart rate zone, showed no significant group differences in FGF2 and VEGF (Table 2).

Stratifying results by baseline $\mathrm{BMI}, \mathrm{VO}_{2 \max }$, and total body fat did not reveal any sub-group effects for VEGF (Table 2). Conversely, the result for FGF2 varied by baseline $\mathrm{VO}_{2 \max }$ and total body fat. Specifically, women with lower baseline physical fitness had an overall lower concentration of FGF2 in the HIGH group (TER 0.94, 95\% CI 0.88, 1.00). However, this result was largely driven by the opposite direction of change at six months $(8.2 \%$ increase in MODERATE group vs. $2.2 \%$ decrease in $\mathrm{HIGH}$ group). At 12 months, the HIGH group had a greater increase in FGF2 from baseline $(8.2 \%$ vs $1.3 \%$ in MODERATE group). Likewise, the statistically significant TER for FGF2 observed among women with greater total body fat at baseline (TER 0.94; 95\% CI 0.88, 1.00) was driven by the opposite direction of change at 6 months (5.8\% increase in MODERATE group vs $2.2 \%$ decrease in HIGH group), while FGF2 showed a greater increase in $\mathrm{HIGH}$ group $(8.5 \%$ vs $4.5 \%$ in MODERATE group).

\section{Discussion}

Growth factors (i.e., FGF2 and VEGF) and their receptors (FGF2R and VEGFR), have been implicated in breast cancer tumorigenesis $[10,11]$, progression $[4,11]$, and metastasis [4]. However, the expression of specific FGF2 and VEGF polymorphisms has also been shown to be protective against developing breast cancer $[12,13]$. In BETA, the greatest increases in FGF2 expression were observed with HIGH volumes of exercise in those with lower baseline physical fitness and higher total body fat whereas, VEGF expression was unaltered.

Our findings may be partially explained by the followup assessment timing and the somewhat differential nature of each growth factor's pro-angio/arteriogenic action. In general, FGF2 and VEGF are important mediators of pro-angiogenic adaptations to repetitive exercise and act to increase tissue capillary density in response to hypoxic and inflammatory signaling [14]. However, it is well known that VEGF signaling is more central to supporting angiogenesis and neovascularization whereas FGF2 signaling plays a larger role in arteriogenesis and the maintenance of mature vessels $[3,15]$. In general, chronically elevated growth factor levels may be considered pathologic - reflective of underlying tumor proliferative processes. However, in the context of exercise, transient growth factor increases can be viewed as normal physiologic hypoxia-driven adaptations - reflective of favorable vascular bed remodeling. While we would have expected to observe a transient increase in levels of these growth factors following the exercise intervention, in the context of the 12-month intervention within BETA, the six-month gap between follow-up assessments may have limited our ability to capture the transient spikes in the expression of these growth factors. Following an acute bout of exercise, the resulting increase in growth factor expression also appears to be transient - elevated after 7-10 days of training and returning to baseline after 28 days $[16,17]$.

In conclusion, high doses of supervised exercise did not impact levels of VEGF or FGF2. Additional mechanisms related to decreased breast cancer risk through altered adipose tissue require investigation.

\footnotetext{
Abbreviations

BETA: Breast cancer and Exercise Trial in Alberta; BMI: Body mass index; FGF2: Fibroblast growth factor-2; ITT: Intention-to-treat; TER: Treatment effect ratio; VEGF: Vascular endothelial growth factor
}

\footnotetext{
Acknowledgements

Calgary Study Coordinators were: Krista Carlson, Sana Fakih, Megan Farris, Quinn Harris, Sarah MacLaughlin, Erica Roberts, and Kristen Simone. Edmonton Study Coordinators were: Natalie Ilkiw, Ciara Kallal, and Dr. Amy Speed Andrews. Assistance with information sessions was provided in Calgary by Drs. Brigid Lynch and Fabiola Aparicio-Ting. Calgary Exercise Trainers were: Carrie Anderson, Alia Bharwani, Shannon Brown, Ashley Cuthbert, Sue Daniel, Julie Gowans, Margo Graham, Erin Korsbrek, Kathleen Kranenburg, Jessica Morrison, Jason Ng, Nicole Slot, Tania White, and Kaila Wright. Edmonton Exercise Trainers were: Arne Anderson, Lisa Belanger, Jennifer Crawford, Cindy Forbes, Alyssa Hindle, Corey Kuzik, Erin McGowan, Mary
} 
Norris, Janel Park, Julianne Symons, Linda Trinh, Stephanie Voaklander and Lynne Wong. Study recruiters were: Jennie Duke, Jasdeep Hayer, Trisha Kelly, Jasmine Lee, and Lilly Mah. Data entry was done by: Sinead Boyle, Barbara Mercer, Carla Quesnel and Trisha Kelly. Data management, including database creation, questionnaire design, data integrity and quality control, was done by: Dr. Steven Szarka, Farit Vakhetov, and Wendy Walroth. Qinggang Wang was responsible for the randomization procedures, sample size calculations and some data verification. The late Dr. Robert C. Millikan was a coinvestigator on this trial and contributed to the study design and methods.

\section{Funding}

This work was supported by a Career Development Award in Prevention (\#703917) from the Canadian Cancer Society Research Institute held by Dr. Brenner. Dr. Friedenreich holds a Health Senior Scholar Award from Alberta Innovates-Health Solutions and is the Alberta Cancer Foundation Weekend to End Women's Cancers Breast Cancer Chair. Dr. Courneya holds a Canada Research Chair in Physical Activity and Cancer.

\section{Availability of data and materials}

The datasets generated during and/or analyzed during the current study are not publicly available but are available from the corresponding author on reasonable request.

\section{Authors' contributions}

DB assisted in data collection, interpretation and wrote the manuscript. YR completed the data analysis and assisted in interpretation and writing of the manuscript. SA assisted in interpretation and writing of the manuscript. CF and $\mathrm{KC}$ were responsible for the design and conduct of the study, data collection as well as writing and critical review of the manuscript. All authors read and approved the final manuscript.

\section{Ethics approval and consent to participate}

This study was approved by the Health Research Ethics Board of Alberta and all participants consented to participate.

\section{Consent for publication}

Not applicable.

\section{Competing interests}

The authors declare that they have no competing interests.

\section{Publisher's Note}

Springer Nature remains neutral with regard to jurisdictional claims in published maps and institutional affiliations.

\section{Author details}

'Department of Oncology, Cumming School of Medicine, University of Calgary, Calgary, Alberta, Canada. ${ }^{2}$ Department of Community Health Sciences, Cumming School of Medicine, University of Calgary, Calgary, Alberta, Canada. ${ }^{3}$ Department of Cancer Epidemiology and Prevention Research, CancerControl Alberta, Alberta Health Services, Holy Cross Centre Room 513C, Box ACB, 2210 - 2nd St. SW, Calgary, Alberta T2S 3C3, Canada. ${ }^{4}$ Faculty of Physical Education and Recreation, University of Alberta, Edmonton, Alberta, Canada.

Received: 1 November 2017 Accepted: 19 April 2018

Published online: 24 June 2019

\section{References}

1. Friedenreich CM, Neilson HK, Lynch BM. State of the epidemiological evidence on physical activity and cancer prevention. Eur J Cancer. 2010;46: 2593-604

2. MCTiernan A, Sorensen B, Yasui $Y$, Tworoger SS, Ulrich CM, Irwin ML, et al. No effect of exercise on insulin-like growth factor 1 and insulin-like growth factor binding protein 3 in postmenopausal women: a 12-month randomized clinical trial. Cancer Epidemiol Biomark Prev. 2005;14:1020-1.

3. Carmeliet P. Mechanisms of angiogenesis and arteriogenesis. Nat Med. 2000;6:389-96

4. Cao R, Ji H, Feng N, Zhang Y, Yang X, Andersson P, et al. Collaborative interplay between FGF-2 and VEGF-C promotes lymphangiogenesis and metastasis. Proc Natl Acad Sci. 2012;109:15894-9.
5. Michailidou K, Hall P, Gonzalez-Neira A, Ghoussaini M, Dennis J, Milne RL et al. Large-scale genotyping identifies 41 new loci associated with breast cancer risk. Nat Genet 2013;45:353-361, 361e351-352.

6. Friedenreich CM, Neilson HK, O'Reilly R, Duha A, Yasui Y, Morielli AR, et al. Effects of a high vs moderate volume of aerobic exercise on adiposity outcomes in postmenopausal women: a randomized clinical trial. JAMA Oncol. 2015;1:766-76.

7. Sun K, Wernstedt Asterholm I, Kusminski CM, Bueno AC, Wang ZV, Pollard JW, et al. Dichotomous effects of VEGF-A on adipose tissue dysfunction. Proc Natl Acad Sci U S A. 2012;109:5874-9.

8. Friedenreich CM, MacLaughlin S, Neilson HK, Stanczyk FZ, Yasui Y, Duha A, et al. Study design and methods for the breast Cancer and exercise trial in Alberta (BETA). BMC Cancer. 2014;14:919.

9. Hermann N, Dreßen K, Schildberg FA, Jakobs C, Holdenrieder S. Methodical and pre-analytical characteristics of a multiplex cancer biomarker immunoassay. World Journal of Methodology. 2014;4:219-31.

10. Beeghly-Fadiel A, Shu X-O, Lu W, Long J, Cai Q, Xiang Y-B, et al. Genetic variation in VEGF family genes and breast Cancer risk: a report from the Shanghai breast Cancer genetics study. Cancer Epidemiol Biomark Prev. 2011;20:33-41.

11. Jain VK, Turner NC. Challenges and opportunities in the targeting of fibroblast growth factor receptors in breast cancer. Breast Cancer Res. 2012; 14:208

12. Rodrigues $\mathrm{P}$, Furriol J, Tormo E, Ballester $\mathrm{S}$, Lluch A, Eroles P. The singlenucleotide polymorphisms +936 C/T VEGF and -710 C/T VEGFR1 are associated with breast cancer protection in a Spanish population. Breast Cancer Res Treat. 2012;133:769-78.

13. Turner N, Grose R. Fibroblast growth factor signalling: from development to cancer. Nat Rev Cancer. 2010;10:116-29.

14. Wagner PD. The critical role of VEGF in skeletal muscle angiogenesis and blood flow. Biochem Soc Trans. 2011;39:1556.

15. Prior BM, Yang $H$, Terjung RL. What makes vessels grow with exercise training? J Appl Physiol. 2004;97:1119-28.

16. Cuevas-Ramos D, Almeda-Valdés P, Meza-Arana CE, Brito-Córdova $G$, Gómez-Pérez FJ, Mehta R, et al. Exercise increases serum fibroblast growth factor 21 (FGF21) levels. PLoS One. 2012;7:e38022.

17. Gustafsson T, Knutsson A, Puntschart A, Kaijser L, Nordqvist SA-C, Sundberg $C$, et al. Increased expression of vascular endothelial growth factor in human skeletal muscle in response to short-term one-legged exercise training. Pflugers Arch. 2002;444:752-9.

\section{Ready to submit your research? Choose BMC and benefit from:}

- fast, convenient online submission

- thorough peer review by experienced researchers in your field

- rapid publication on acceptance

- support for research data, including large and complex data types

- gold Open Access which fosters wider collaboration and increased citations

- maximum visibility for your research: over $100 \mathrm{M}$ website views per year

At BMC, research is always in progress.

Learn more biomedcentral.com/submissions 